\title{
EFFECTS OF VALSARTAN AND AMLODIPINE ON PROINFLAMMATORY CYTOKINES SERUM CONCENTRATIONS IN SALT LOADED SPONTANEOUSLY HYPERTENSIVE RATS
}

\author{
Kalina Gjorgjievska ${ }^{1}$, Dejan Trajkov ${ }^{2}$, Dimche Zafirov ${ }^{1}$, Aleksandar Petlichkovski ${ }^{2}$, Krume Jakjovski $^{1}$, \\ Emilija Atanasovska ${ }^{1}$, Marija Petrusevska ${ }^{1}, K_{\text {Kristina Pavlovska }}{ }^{1}$, Olgica Sibinovska ${ }^{2}$, \\ Dragica Zendelovska ${ }^{1}$
}

\begin{abstract}
${ }^{I}$ Department of Preclinical and Clinical Pharmacology and Toxicology, University "Ss. Cyril \& Methodius" Medical Faculty, Skopje, Macedonia, ${ }^{2}$ Institute of Immunobiology and Human Genetics, Medical Faculty, University Ss. Cyril and Methodius, Skopje, Macedonia
\end{abstract}

Background: Inflammation' as measured by proinflammatory cytokines (interleukin [IL]-1' IL-6 and tumor necrosis factor $\alpha[\mathrm{TNF} \alpha])^{\prime}$ is implicated in the development and maintenance of hypertension in patients 1 and animal experimental models2-5. Salt loading as method for hypertension progression is extensively investigated in rats6-8. In this study we have studied the effect of salt loading on cytokine serum concentrations in SHR rats and evaluated possible immunomodulatory properties of valsartan and amlodipin monotherapy and combination.

Materials and methods: 80 eight-week old SHR rats were used. Rats were divided into 2 groups: control group (SHRC ' $\mathrm{n}=16)$ given tab water ad libitum and a salt loaded group $(\mathrm{n}=64)$ in which tab water was replaced with $\mathrm{NaCl}$ solution (1\%) given ad libitum for the complete course of the study. Salt loaded group after 12 weeks was divided in 3 treatment groups (SHRVal- valsartan as $10 \mathrm{mg} / \mathrm{kg} / \mathrm{b}$. w.' SHRAmlo- amlodipin as $5 \mathrm{mg} / \mathrm{kg} / \mathrm{b}$. w. 'SHRVal/Amlo - $5 \mathrm{mg} / \mathrm{kg}$ valsartan $+2.5 \mathrm{mg} / \mathrm{kg}$ amlodipin per b. w.) and salt loaded control (SHRNaCl). After 12 weeks of drug treatment ' serum TNF $\alpha$ ' IL-1 and IL-6 concentrations were measured with commercially available enzyme-linked immunosorbent assay kits (Cusabio Biotech Co. Ltd) according to the manufacturer's instructions. In this study lipopolysaccharide (LPS) challenge was not used. Differences between certain time points and groups were analyzed with the Student's t-test for dependent and independent parameters' Kruskal Wallis ANOVA and Mann-Whitney U-test. Values of $\mathrm{p}<0.05$ were considered to be statistically significant.

Results: As shown in Table 1' by week 24 of the study' SHRNaCl presented higher IL-6 ' TNF $\alpha$ and IL-1 serum concentrations in comparison to SHRC group. Valsartan/amlodipin combination therapy effectively reduced IL-6 and TNF $\alpha$ serum concentrations in comparison to SHRNaCl group. There was no significant decrease in IL-1 concentrations in all drug treated groups in comparison to measured IL-1 concentrations in salt loaded control.

Conclusions: Salt loading with $\mathrm{NaCl}$ solution (1\%) in SHR rats for a period of 24 weeks had led to significant increase of measured proinflammatory cytokines. Valsartan and amlodipine effectively modulated IL- 6 and TNF $\alpha$ levels. Drugs complementary mechanism had additive beneficial effect on proinflamatory cytokines. 